\title{
The Wilson loop from a Dyson equation
}

\author{
M. Pak and H. Reinhardt \\ Institut für Theoretische Physik \\ Auf der Morgenstelle 14 \\ D-72076 Tübingen \\ Germany
}

\begin{abstract}
The Dyson equation proposed for planar temporal Wilson loops in the context of supersymmetric gauge theories is critically analysed thereby exhibiting its ingredients and approximations involved. We reveal its limitations and identify its range of applicability in non-supersymmetric gauge theories. In particular, we show that this equation is applicable only to strongly asymmetric planar Wilson loops (consisting of a long and a short pair of loop segments) and as a consequence the Wilsonian potential can be extracted only up to intermediate distances. By this equation the Wilson loop is exclusively determined by the gluon propagator. We solve the Dyson equation in Coulomb gauge for the temporal Wilson loop with the instantaneous part of the gluon propagator and for the spatial Wilson loop with the static gluon propagator obtained in the Hamiltonian approach to continuum Yang-Mills theory and on the lattice. In both cases we find a linearly rising color potential.
\end{abstract}

PACS numbers: 11.10.Ef,12.38.Aw,12.38.Lg

Keywords: Wilson Loop, Confinement, Coulomb gauge, Hamiltonian approach

\section{INTRODUCTION}

The Wilson loop is a quantity of central interest in Yang-Mills theory. The temporal Wilson loop is related to the static potential between infinitely heavy color sources and represents the order parameter of confinement: An area law in the temporal Wilson loop corresponds to a linearly rising potential. Furthermore the potential $V(L)$ extracted from a rectangular temporal Wilson loop of spatial extension $L$ is the ground state energy of a pair of an infinitely heavy quark and antiquark at separation $L$. As discussed in detail in Ref. [1] this "Wilsonian" potential $V(L)$ is not the fundamental color confining potential $V_{C}(L)$, but rather the residual potential that survives color screening by vacuum polarization effects, i.e. $V(L)$ contrary to $V_{C}(L)$ contains the back reaction of the Yang-Mills vacuum to the presence of the infinitely heavy color sources. Obviously, the screening of color charges will lower the energy so that $V(L)<V_{C}(L)$. Therefore, a linearly rising fundamental potential $V_{C}(L)$ does not necessarily imply an area law in the corresponding Wilson loop. The fundamental color potential $V_{C}(L)$ can be explicitly isolated in Coulomb gauge, Ref. [2], and it was shown in Ref. [3] that the so-called "Coulomb string tension" $\sigma_{C}$ extracted from $V_{C}(L)$ is indeed an upper bound to the Wilsonian string tension $\sigma$.

It is still a big challenge to understand the color confinement mechanism in the continuum Yang-Mills theory, which requires to prove the area law for the Wilson loop. In recent years several approaches to continuum Yang-Mills theory have been intensively pursued. Among others there are the Dyson-Schwinger approach in Landau gauge, Ref. [4], and more recently also in Coulomb gauge, Ref. [5], and a variational solution of the Yang-Mills Schrödinger equation in Coulomb gauge, Ref. [6, 7, 8, [9, 10]. These approaches have given support for the Gribov-Zwanziger confinement scenario, Ref. [11, 12]. What is, however, missing in these approaches is the explicit non-perturbative evaluation of the Wilson loop, showing the emergence of the area law. In the continuum theory the calculation of the Wilson loop is rendered complicated due to the path ordering. In the context of supersymmetric Yang-Mills theory a Dyson type of integral equation was proposed for the temporal Wilson loop, which sums all planar ladder (or rainbow) diagrams, Ref. 13]. This equation takes care of the path ordering, at least in an approximate fashion, and was recently also applied to the temporal Wilson loop in ordinary (non-supersymmetric) Yang-Mills theory, Ref. [14]. Here the only input into this Dyson equation is the gluon propagator, which is gauge dependent. In Ref. 14 the gluon propagator was found by solving the Dyson-Schwinger equations of Yang-Mills theory in Landau gauge in the rainbow-ladder approximation. In the present paper we will critically review the Dyson equation for the Wilson loop, work out its ingredients and shortcomings, and apply it to Yang-Mills theory in Coulomb gauge. We study both the temporal and spatial Wilson loop.

The organization of the paper is as follows: In the next section we give a short heuristic derivation of the Dyson equation for the Wilson loop proposed in Ref. [13] and critically analyse the ingredients, the approximations involved and exhibit its range of validity. In Sect. III we solve this equation for the temporal Wilson loop in Coulomb gauge assuming an instantaneous, temporal gluon propagator. In Sect. IV the Dyson equation for the Wilson loop with

Typeset by REVTEX 
arbitrary gluon propagators is converted to a one-dimensional Schrödinger equation following Ref. [13]. In Sect. V we use this Schrödinger equation to calculate the spatial Wilson loop in the Hamiltonian approach to Yang-Mills theory in Coulomb gauge. Finally our conclusions are given in Sect. VI.

\section{THE DYSON EQUATION FOR THE WILSON LOOP}

Below we briefly sketch the derivation of the Dyson equation for the Wilson loop thereby exhibiting the approximations involved and working out its limitations.

Consider the Wilson loop integral

$$
\langle W(\mathcal{C})\rangle=\left\langle\frac{1}{d_{r}} \operatorname{tr} \mathcal{P} \exp \left[-g \oint_{\mathcal{C}} d x_{\mu} A_{\mu}(x)\right]\right\rangle
$$

defined as expectation value via a functional

$$
\langle\ldots\rangle=\frac{\int D A_{\mu} \ldots e^{-S[A]}}{\int D A_{\mu} e^{-S[A]}}
$$

Here $\mathcal{C}$ is a closed loop in Euclidean space, $d_{r}$ is the dimension of the group representation and $\mathcal{P}$ denotes path ordering along this loop. Furthermore $A_{\mu}=A_{\mu}^{a} T_{a}$ is the algebra valued gauge field (referred to as gluon field) with $T_{a}$ being the anti-hermitean generators of the gauge group and $S[A]$ is the action of the underlying gauge theory. In leading order perturbation theory only the quadratic part of the action is kept

$$
S[A]=\frac{1}{2} \int d^{d} x d^{d} y A_{\mu}(x) D_{\mu \nu}^{-1}(x, y) A_{\nu}(y),
$$

with $D_{\mu \nu}(x, y)$ being the bare gluon propagator and $d$ being the number of space-time dimensions, and path ordering can be ignored, yielding for the Wilson loop

$$
W(\mathcal{C})=\exp \left[-\frac{g^{2}}{2} C_{2} I(\mathcal{C})\right]=1-\frac{g^{2}}{2} C_{2} I(\mathcal{C})+\cdots
$$

where

$$
I(\mathcal{C})=\oint_{\mathcal{C}} d x_{\mu} \oint_{\mathcal{C}} d y_{\nu} D_{\mu \nu}(x, y)
$$

and

$$
T_{a} T_{a}=-\mathbb{1}_{(d)} C_{2}
$$

is the quadratic Casimir operator. For the gauge group $S U(N)$ we have $C_{2}=\frac{N^{2}-1}{2 N}$. Obviously, the perturbative gluon propagator $D(k) \sim \frac{1}{k^{2}}$ cannot give rise to an area law.

Consider now a temporal planar trapezoidal Wilson loop $W=W(S, T ; L)$ with two parallel temporal sides of length $L$ and $T$, separated by a spatial distance $L$, see Fig. 1. This loop consists of four straight paths. Accordingly, the loop integral (5) receives 16 contributions. In the limit $S, T \gg L$ the dominant contribution comes from the two temporal paths (of length $T$ and $S$, respectively). Keeping only these two paths and ignoring the contributions where both integrals in (5) run along the same temporal path, this loop integral reduces to

$$
I(\mathcal{C})=-2 \int_{0}^{S} d x_{0} \int_{0}^{T} d y_{0} D_{00}\left(\left(x_{0}-y_{0}\right)^{2}+L^{2}\right)
$$

The diagram corresponding to this contribution to the Wilson loop is shown in Fig. 2(a). Since the gluon propagator (exact or perturbative) drops off for large distances one may argue that for $S, T \gg L$ the contribution to the loop integral $I(\mathcal{C})$ (5) with gluon lines connecting the spatial paths as shown in Fig. 2(b) are subleading compared to the contribution from the temporal paths shown in Fig. 2(a). Then in the standard way one may resum all the ladder 


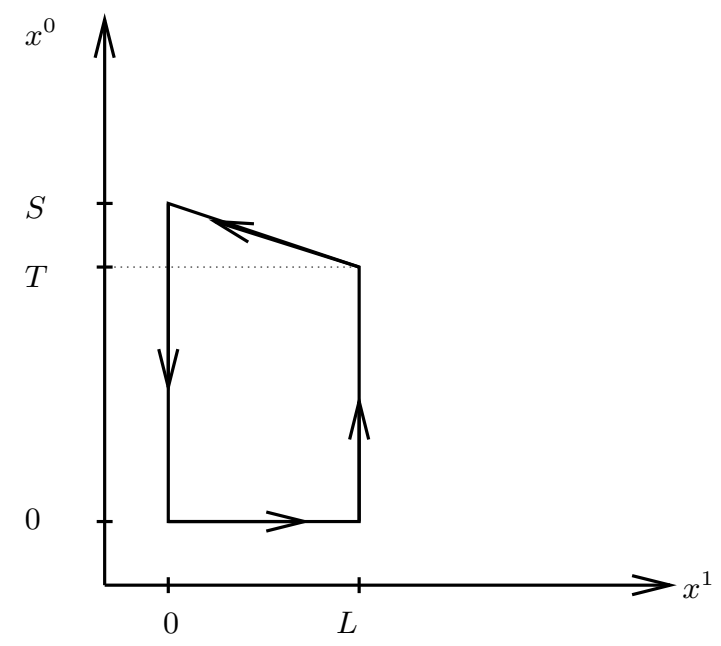

FIG. 1: Trapezoidal temporal Wilson loop.

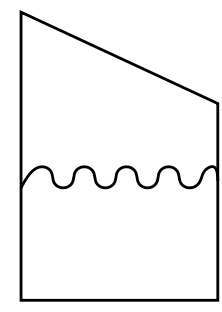

$(a)$

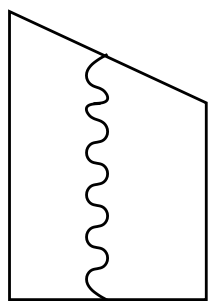

$(b)$

FIG. 2: (a) Processes involved and (b) processes ignored in the Dyson equation (8) illustrated in Fig. 3 .

diagrams with gluon lines connecting the two temporal paths shown in Fig. 3(a) by a Dyson equation with kernel given by the gluon propagator. This yields the integral equation originally proposed in the context of supersymmetric gauge theories, Ref. [13], which is illustrated in Fig. 3(b) and given by

$$
W(S, T ; L)=1+g^{2} C_{2} \int_{0}^{S} d s \int_{0}^{T} d t D\left((x(s)-s(t))^{2}\right) W(s, t ; L),
$$

where

$$
D\left((x(s)-x(t))^{2}\right)=\dot{x}_{\mu}^{-}(s) D_{\mu \nu}(x(s), x(t)) \dot{x}_{\nu}^{+}(t)
$$

and $x_{\mu}^{ \pm}(s)$ denotes a parametrization of the two temporal paths of the Wilson loop (see the Appendix for an explicit realization of these paths). Obviously, the summation of the ladder diagrams by the Dyson equation remains valid when the full gluon propagator is used and does not lead to any double counting.

The above given sketch of the derivation of the Dyson equation clearly exhibits the limitations of this equation:

1. The Dyson equation (8) can be only applied to strongly asymmetric loops consisting of two opposite long (above temporal) and two opposite short (above spatial) paths. Otherwise it does not make sense to include one pair of paths (Fig. 2(a)) in the contour integration (5) and partially sum their ladder diagrams while neglecting the contour integrals of the other pair of paths (Fig. 2(b)) and their ladders. It is, however, not necessary that the Wilson loop is a temporal one. Eq. (8) can be equally well applied to strongly asymmetric spatial loops. Due to the limitation to asymmetric loops the static potential extracted from the solution of Eq. (8) will be accessible only for spatial distances $L \ll T$. In particular, the limit $L \rightarrow \infty$ is not accessible by this equation.

2. The solutions of the Dyson equation (8) obviously satisfy the boundary conditions

$$
W(S, T=0 ; L)=1, W(S=0, T ; L)=1 .
$$




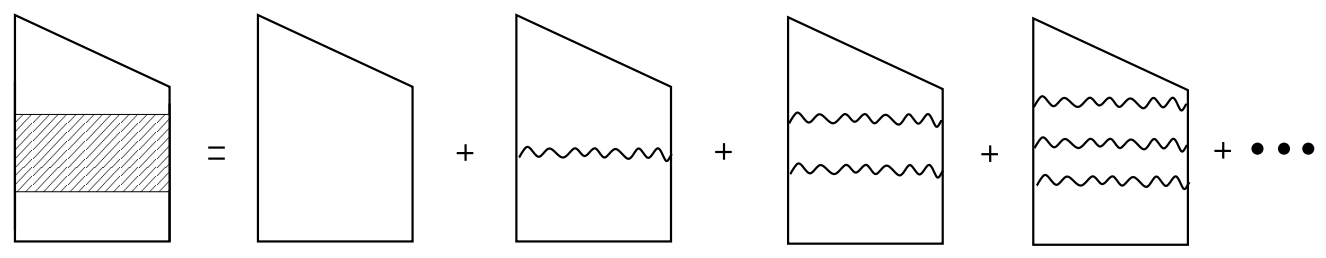

(a)

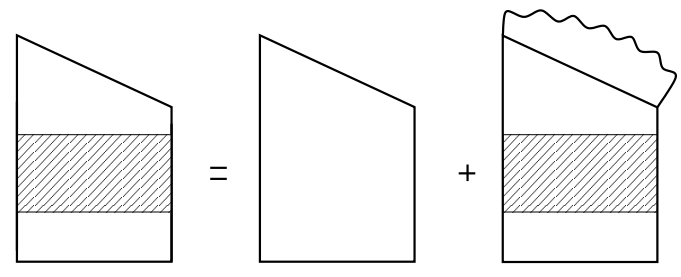

(b)

FIG. 3: Graphical illustration to (a) the Dyson series for the Wilson loop and (b) the Dyson equation (8).

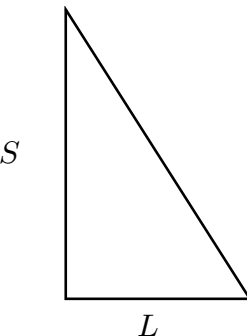

$T=0$

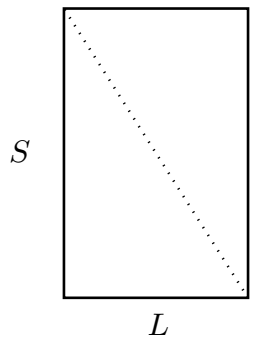

$T=S$

(a)

(b)

FIG. 4: (a) Triangle and (b) rectangular shaped loops representing the Wilson loop $W(S, T=0 ; L)$ and $W(S, S ; L)$, see text.

However, for $T=0$ or $S=0$ the trapezoidal loop degenerates to a triangle shaped loop, shown in Fig. 4 (a) and the Wilson loop of the triangle shaped contour is certainly not one. The wrong boundary value (10) comes with no surprise since the limit $T=0$ or $S=0$ contradicts the assumption $S, T \gg L$ inherent in Eq. (8). The wrong boundary value (10) has consequences for the Wilsonian potential extracted from the rectangular Wilson loop $W(S=T, T ; L)$ shown in Fig. 4(b). Assume the solution of the Dyson equation (8) yields asymptotically indeed an area law as expected for Yang-Mills theory. Due to the boundary value (10), $W(S=T, T ; L)$ misses the contribution from half of its enclosed area (i.e. from the triangle shown in Fig. [(a)). If the remaining triangle has to account for the full rectangle the string tension has to be doubled. Consequently we expect from the solution of the Dyson equation (8) the double of the true string tension.

3. In the Dyson equation (8) the only remnant from the representation of the gauge group is the quadratic Casimir (6). Therefore, the Wilson loop obtained as a solution of Eq. (8) will show strict Casimir scaling. It is known, however, that Casimir scaling occurs only in the intermediate distance regime, Ref. [15]. At large distances the string tension survives only for the group representations with odd $N$-ality ${ }^{1}$ while the charges of the even $N$-ality representations are screened. Thus again we find that the Dyson equation (8) can be appropriate only up to some intermediate distances and is bound to fail for large (spatial) distances.

4. The Wilson loop, per se, is gauge invariant while the right hand side of the Dyson equation (8) is gauge dependent

${ }^{1} N$-ality characterizes the representation of the center in an irreducible representation of the group. The center of $S U(N)$ is $Z(N)$, consisting in the fundamental representation of the $N$ group elements $z_{n}=\exp \left(i \frac{2 \pi n}{N}\right) \mathbb{1}_{(N)}, n=0,1,2, \ldots, N-1$. Multiplication of a group element $g$ of an irreducible representation with $N$-ality $k$ by the center element $z_{n}$ implies the multiplication of $g$ by the factor $\exp \left(i \frac{2 \pi n}{N} k\right)$. 
through the gluon propagator. In fact, the gluon propagator is non-vanishing only after the gauge has been fixed. Thus, Eq. (8) will yield different results for the Wilson loop in different gauges.

5. Finally, let us also mention that (except for the temporal Wilson loop in Coulomb gauge, see below) the right hand side of the Dyson equation (8) is not renormalization group invariant, while the Wilson loop is.

From the above analysis it is clear that the Dyson equation (8) can be applied only to strongly asymmetric loops with two different length scales and that the smaller length scale has to be restricted from above to intermediate distances. The limitations of the Dyson equation (8) revealed above will manisfest themselves in the applications of this equation to be given below.

\section{THE TEMPORAL WILSON LOOP IN COULOMB GAUGE}

Since the Dyson equation (8) for the Wilson loop is gauge dependent via the gluon propagator it is preferable to use so-called "physical" gauges, for which the gauge constraint and Gauss' law can be explicitly resolved, leaving a gauge fixed theory of the physical degrees of freedom. Such a gauge is the Coulomb gauge

$$
\partial \cdot \boldsymbol{A}=0 .
$$

In addition this gauge has the advantage that the quantity $g A_{0}$ is renormalization group invariant, which is not the case for covariant gauges like Landau gauge. The Dyson equation (8) for the temporal Wilson loop contains only the temporal component of the gluon propagator

$$
g^{2} D_{00}^{a b}(x, y)=\left\langle g A_{0}^{a}(x) g A_{0}^{b}(y)\right\rangle .
$$

Therefore in Coulomb gauge the Dyson equation (8) is renormalization group invariant and thus independent of the regularization and renormalization scheme. Let us therefore investigate the Dyson equation in Coulomb gauge. In Coulomb gauge the temporal gluon propagator has the structure [1]

$$
g^{2} D_{00}^{a b}(x, y)=-\delta^{a b} V_{C}(|\boldsymbol{x}-\boldsymbol{y}|) \delta\left(x^{0}-y^{0}\right)+P^{a b}(x, y) .
$$

Here $V_{C}(|\boldsymbol{x}-\boldsymbol{y}|)$ is the so-called non-Abelian Coulomb potential, which is long-range and presumably responsible for color confinement. It describes anti-screening of color charges, while $P(x, y)$, also being long-range, describes ordinary screening. The latter is responsible for the breaking of the color flux string between the external quarks when dynamical quarks are present. Both terms have been calculated in perturbation theory [1, 17, 18, 19]. The non-Abelian Coulomb potential $V_{C}(|\boldsymbol{x}-\boldsymbol{y}|)$ was also non-perturbatively calculated both in the Hamiltonian approach to continuum Yang-Mills theory [7, 9] and on the lattice [20, 21, 22]. At small distance it behaves like the ordinary Coulomb potential $\sim 1 / r$, while it rises linearly at large distance $\sim \sigma_{C} r$ with a coefficient $\sigma_{C}$, referred to as Coulomb string tension, which is somewhat larger than the Wilsonian string tension $\sigma$ extracted from the (temporal) Wilson loop $\left(\sigma_{C} \sim 1.5 \sigma\right.$ Ref. [22] $)$.

Since we are mainly interested in the infrared properties of the Wilson loop let us keep here the instantaneous (confinement relevant) part of the gluon propagator (13) only. This forces us to consider rectangular Wilson loops

$$
\bar{W}(T ; L):=W(T, T ; L),
$$

for which the Dyson equation (8) reduces to

$$
\bar{W}(T ; L)=1-C_{2} V_{C}(L) \int_{0}^{T} d t \bar{W}(t ; L) .
$$

This equation can be converted into the differential equation

$$
\frac{d}{d T} \bar{W}(T ; L)=-C_{2} V_{C}(L) \bar{W}(T, L)
$$

with the boundary condition

$$
\bar{W}(T=0 ; L)=1,
$$


which is, contrary to the boundary condition of the general case (10), indeed the correct boundary condition (the area enclosed by the loop vanishes for $T=0$ ). The solution of Eqs. (16), (17) or equivalently of (15) is given by

$$
\bar{W}(T ; L)=\exp \left(-C_{2} V_{C}(L) T\right) .
$$

Since the non-Abelian Coulomb potential rises linearly at large distances we have correctly obtained an area law, however, with the Wilsonian string tension $\sigma$ replaced by the Coulomb string tension $\sigma_{C}>\sigma$. This is expected due to the neglect of the non-instantaneous part $P(x, y)$ of the gluon propagator (13), which screens the non-Abelian charge of the static quarks represented by the temporal Wilson lines. Within the instantaneous approximation used for the gluon propagator (13) $\left(g^{2} D_{00}(x) \rightarrow-V_{C}(\boldsymbol{x}) \delta\left(x^{0}\right)\right)$ Eq. (18) is the correct result. It is clear why in the present case the Dyson equation (8) yields the correct Wilson loop: The processes neglected in the Dyson equation (8) (see Eq. (2) (b)) do not exist for an instantaneous gluon propagator.

\section{EXTRACTING THE STATIC POTENTIAL}

The charm of the Dyson equation (8) for the Wilson loop is its simplicity. It can be reduced to a one-dimensional Schrödinger equation and from the corresponding ground state energy the static potential can be extracted [13]. Differentiation of Eq. (8) with respect to $S$ and $T$ yields the differential equation

$$
\frac{\partial^{2} W(S, T ; L)}{\partial S \partial T}=g^{2} C_{2} D\left(L^{2}+(S-T)^{2}\right) W(S, T ; L),
$$

which together with the boundary condition (10) is equivalent to the integral equation (8) but easier to solve. By introducing the variables

$$
r=\frac{S-T}{L} \quad, \quad R=\frac{S+T}{L} \quad, \quad \frac{\partial^{2}}{\partial S \partial T}=\frac{1}{L^{2}}\left(\frac{\partial^{2}}{\partial R^{2}}-\frac{\partial^{2}}{\partial r^{2}}\right)
$$

this equation is separable [13]. With the notation $W(R, r):=W(S, T ; L)$ its solution can be expressed as

$$
W(R, r)=\sum_{n} \varphi_{n}(r)\left(c_{n}^{+} \exp \left(\Omega_{n} R / 2\right)+c_{n}^{-} \exp \left(-\Omega_{n} R / 2\right)\right)
$$

where the $\varphi_{n}(x)$ satisfy the 1-dimensional Schrödinger equation

$$
\left[-\frac{d^{2}}{d r^{2}}+U(r)\right] \varphi_{n}(r)=-\frac{\Omega_{n}^{2}}{4} \varphi_{n}(r)
$$

with the potential

$$
U(r)=-g^{2} C_{2} L^{2} D\left(L^{2}\left(1+r^{2}\right)\right)
$$

and the constants $c_{n}^{ \pm}$have to be chosen such that $W$ satisfies the boundary conditions (10), which in the variables (20) read $W(R, r=R)=W(R, r=-R)=1$ for all $R$. Since the potential is symmetric $U(-r)=U(r)$ the eigenfunctions $\varphi_{n}(r), n=0,1,2, \ldots$ have definite parity $\varphi_{n}(-r)=(-)^{n} \varphi_{n}(r)$ and the boundery condition implies that $c_{n}^{ \pm}=0$ for $n$-odd. By the symmetry of (21) the $\Omega_{n}$ can be chosen to be positive definite without loss of generality.

We are interested in the Wilson loop $W(S, T ; L) \equiv W(R, r)$ for $L \ll S=T \rightarrow \infty$, i.e. $r=0, R \rightarrow \infty$, which is related to the static potential by

$$
V(L)=-\lim _{T \rightarrow \infty} \frac{1}{T} \ln W(T, T ; L) .
$$

In this limit and for $\Omega_{n}>0$ the Wilson loop is dominated by the first term in (21) with the largest $\Omega_{n}$ for which $\varphi_{n}(0) \neq 0$, which will usually correspond to the ground state $n=0$ of (22)

$$
\left.W(T, T ; L)\right|_{T \gg L}=W(R \rightarrow \infty, r=0) \approx \varphi_{0}(0) e^{\Omega_{0} R / 2} .
$$

We thus obtain for the static potential (24)

$$
V(L)=-\frac{\Omega_{0}(L)}{L}+\text { const } .
$$

Thus, to find the static Wilsonian potential we have to solve the Schrödinger equation (22) for its ground state as a function of $L$. As we have discussed above the validity of the Dyson-Schwinger equation (8) and thus of the expression (26) for the potential is restricted to not too large $L$. The precise range of validity will depend on the scale set by the gluon propagator which defines the potential $U(r ; L)$ given in (23). This will be seen in the results. 


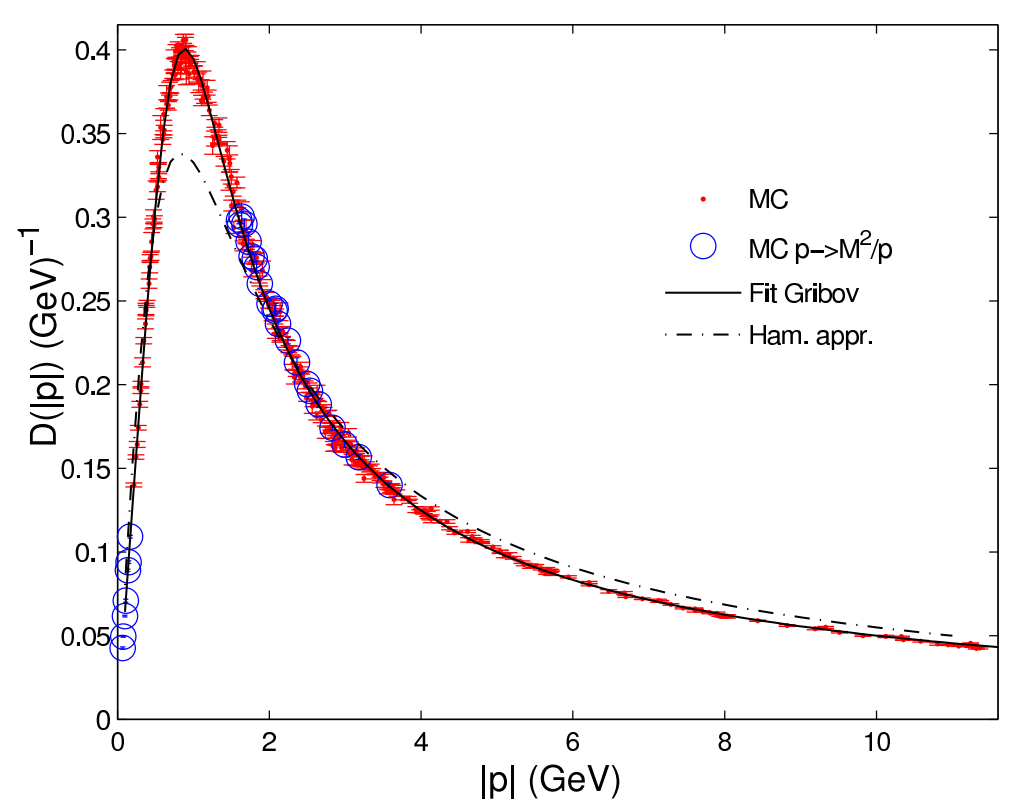

FIG. 5: The gluon energy $\omega(k)$. Data points show the lattice results of Ref. [23]. The full curve is the fit of the lattice data by Gribov's formula (30) while the dashed curve is the result of the variational calculation [9].

\section{SPATIAL WILSON LOOP IN $D=3+1$ COULOMB GAUGE}

At zero temperature, due to $O(4)$ symmetry, the Wilsonian potential can be extracted from either the spatial or temporal Wilson loops. In the Hamiltonian approach, the spatial loop is, however, more easily accessible than the temporal loop, once the vacuum wave functional has been determined. This is because the temporal Wilson loop requires also the time-evolution of the vacuum wave functional. As is clear from the discussion given in Sect. 2 the Dyson equation (8) can be equally well applied to spatial Wilson loops provided the loops are chosen asymmetrically with pairwise long and short paths. We are interested in the spatial Wilson loop in the Hamiltonian approach to Yang-Mills theory in Coulomb gauge 7, 8, 9]. In this case the expectation value is still given by Eq. (2) where the functional integration runs now over the transversal spatial components of the gauge field only and the action $S[A]$ is defined by

$$
e^{-S[A]}=J(A)|\psi[A]|^{2} .
$$

Here $J(A)$ is the Faddeev-Popov determinant and $\psi[A]$ is the vacuum wave functional. In the approach of Ref. 7] the variational ansatz for the wave function $\psi[A]$ is such that the action functional defined by (27) has the form

$$
S[A]=\int d^{3} x d^{3} y A_{i}^{a}(\boldsymbol{x}) \omega(\boldsymbol{x}, \boldsymbol{y}) A_{i}^{a}(\boldsymbol{y})
$$

so that the static (spatial) gluon propagator is given by

$$
D_{i j}^{a b}(\boldsymbol{x}, \boldsymbol{y})=\left\langle A_{i}^{a}(\boldsymbol{x}) A_{j}^{b}(\boldsymbol{y})\right\rangle=\delta^{a b} \frac{1}{2} t_{i j}(\boldsymbol{x}) \omega^{-1}(\boldsymbol{x}, \boldsymbol{y}) .
$$

Here $\omega(\boldsymbol{x}, \boldsymbol{y})$ is a variational kernel, which is found by minimizing the vacuum energy density, and $t_{i j}(\boldsymbol{x})$ is the projector to transversal gauge fields. The result of the variational calculation is confirmed by a lattice calculation of the static gluon propagator [23], see Fig. 5. In the infrared region, which is responsible for the string tension, the variational result almost perfectly matches the lattice data. There are only small deviations in the intermediate momentum regime (see Fig. 5), which shows some dependence on the single remaining undetermined renormalization parameter [24]. Possibly, these deviations result partly from the neglect of the three-gluon vertex. One could perhaps fine-tune the remaining renormalization parameter to get a better agreement with the lattice data in the intermediate momentum regime. However, this region is irrelevant for the asymptotic behaviour of the Wilson loop. The lattice data for the kernel $\omega(k)$ can be nicely fitted by Gribov's formula [1]

$$
\omega(k)=\sqrt{k^{2}+\frac{M^{4}}{k^{2}}},
$$




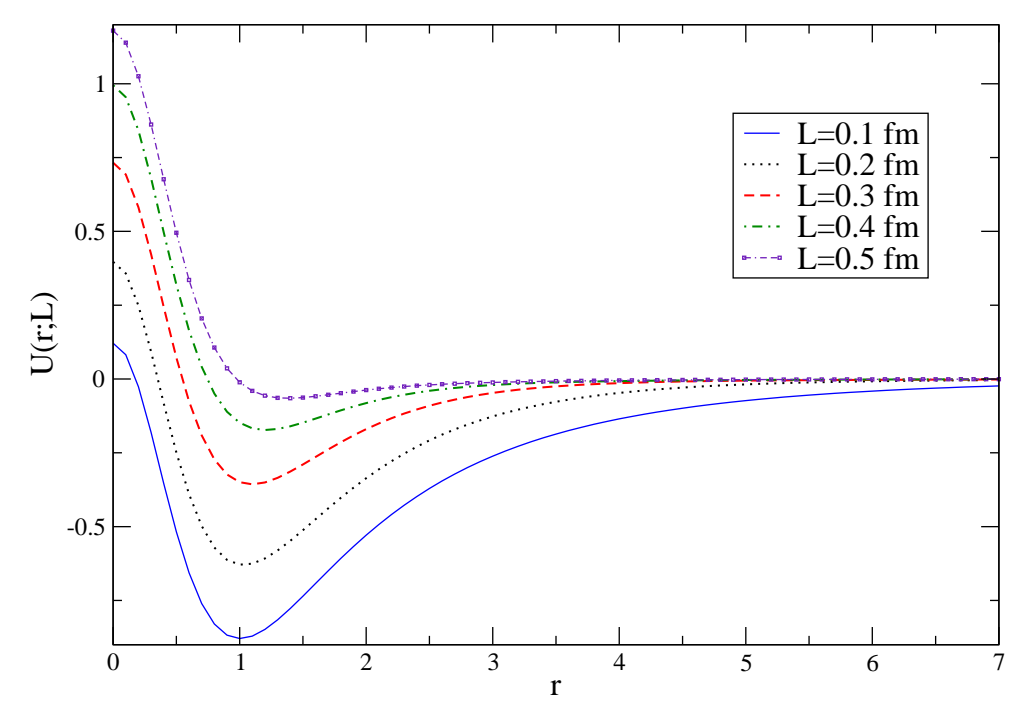

FIG. 6: The potential $U(r ; L)$ 23) for several distances $L$.

where $M$ is a mass scale which, in principle, is determined by the string tension. The fit to the lattice data yields [23]

$$
M \approx 880 \mathrm{MeV} \approx 2 \sqrt{\sigma} .
$$

We will use the static gluon propagator defined by Eqs. (29), (30), (31) in the Dyson equation (8) (i.e. in the potential (23) of the Schrödinger equation (22) ) to calculate the spatial Wilson loop in the Hamiltonian approach in Coulomb gauge.

When the expression (30) is inserted for the (static) propagator $\bar{D}(k)=(2 \omega(k))^{-1}$ the resulting integrals are UVdivergent (see Eq. (A9) in the Appendix). The reason is that Gribov's formula does not include the anomalous dimension of the gluon propagator. The logarithmic momentum dependence induced by the anomalous dimensions is difficult to see on the lattice. In the Hamiltonian approach to the continuum theory [6, 7], which focuses on the infrared physics, the anomalous dimension escapes due to the particular variational ansatz chosen for the vacuum wave functional, which leaves out the three-gluon vertex and thus the gluon loop contribution to the gluon self-energy. The latter is given in this approach by the ghost loop, which dominates in the infrared. The anomalous dimension of the static gluon propagator was studied in Ref. 25]. Including the anomalous dimension the UV-term in Gribov's formula has to be modified to

$$
\omega(k)=\sqrt{k^{2}\left(1+a \ln ^{\gamma} \frac{k}{M}\right)^{2}+\frac{M^{4}}{k^{2}}}
$$

where the anomalous dimension (of the gluon propagator) is given by $\gamma=\frac{3}{11}[25]$. The parameter $a$ depends on $M$. Since the only effect of the anomalous dimension is to make the integrals (A9) UV-convergent we will choose a small value of $a=0.10$. The results do not depend on the precise value of the parameter $a$. Let us stress that the IR-behaviour of the gluon propagator is independent of the anomalous dimension $\gamma$. Fig. 7 shows the potential $U(r ; L)$ (23) calculated from the static propagator (29) in Coulomb gauge with $\omega(k)$ given by Eq. (32) for various spatial distances $L$. $U(r ; L)$ has the form of a double well centered at $r=0$. The dip in the potential, necessary for the formation of a bound state, flattens as $L$ increases and vanishes for $L \geq 0.5 \mathrm{fm}$. However, the bound state disappears already for $L \approx 0.35 \mathrm{fm}$. The Wilson potential can then no longer be extracted from the "ground state energy" $\Omega_{0}(L)$. This limits the use of the Schrödinger equation (22) to rather small distances.

The Wilsonian potential $V(L)$ obtained from the ground state eigenvalue $\Omega_{0}(L)$ via Eq. (26) is shown in Fig. 7 as a function of the spatial distance $L$. The potential behaves like the ordinary Coulomb potential at small distances (below $0.15 \mathrm{fm}$ ) and rises linearly in an intermediate distance regime between 0.15 and $0.35 \mathrm{fm}$. At larger distances $L$ the Schrödinger equation (22) has no bound state and thus Eq. (26) ceases to be applicable. The linear rise in the 

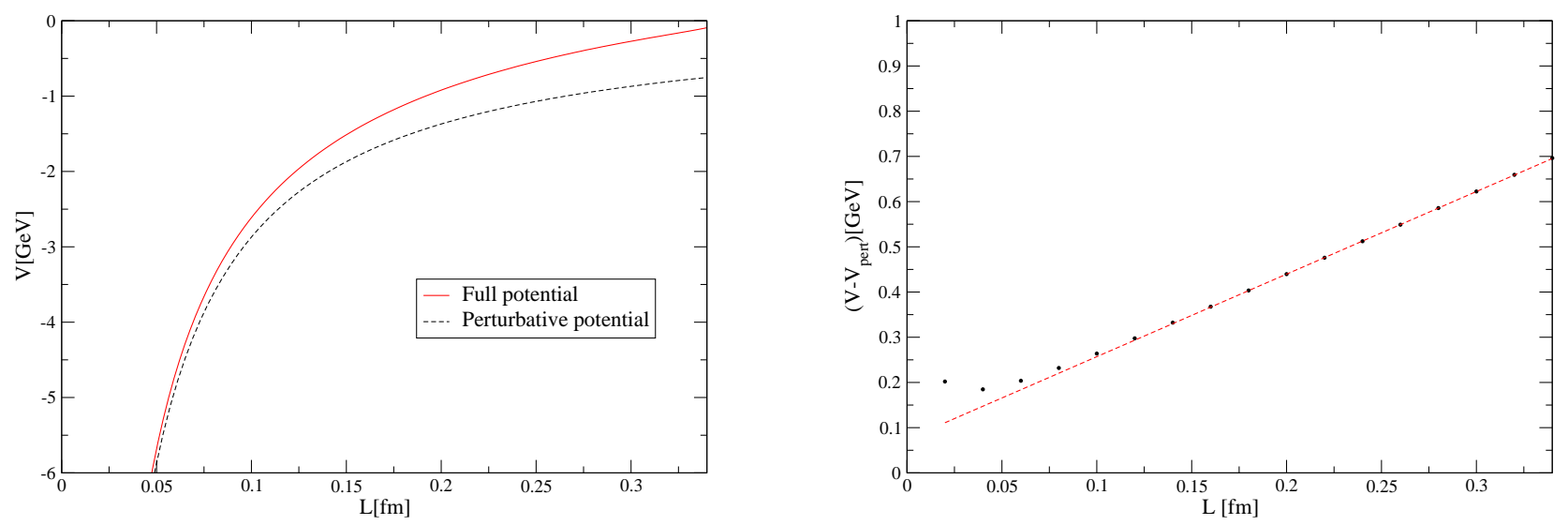

FIG. 7: Left panel: The full static quark potential $V(L)$ obtained from the full propagator (32) and the perturbative potential $V_{\text {pert }}(L)$ obtained from the perturbative propagator (33). Right panel: The full potential minus its perturbative part.

potential is clearly exhibited when the perturbative potential $V_{\text {pert }}(L)$, calculated with the perturbative gluon energy

$$
\omega_{\text {pert }}(k)=|\boldsymbol{k}|\left(1+a \ln ^{\gamma} \frac{k}{M}\right)
$$

is subtracted from the full potential, as shown in Fig. 7 Except for the very small distance regime, which is subject to numerical inaccuracies, the potential void of its perturbative part can be nicely fitted by a linear function (see Fig. 7)

$$
V(L)-V_{\text {pert }}(L)=c+\sigma L,
$$

from which we extract the string tension

$$
\sigma \approx(600 \mathrm{MeV})^{2} .
$$

The string tension is about twice its input value of $\sigma=(440 \mathrm{MeV})^{2}$ used to fix the scale. This is in accord with our remark given in Sect. 2.

\section{SUMMARY AND CONCLUSIONS}

We have studied the Dyson equation for the Wilson loop proposed in Ref. [13] in the context of supersymmetric gauge theory and applied in Ref. [14] to ordinary Yang-Mills theory in Landau gauge. We have examined the approximations involved and exhibited its range of applicability. We have shown that this equation can be applied only to strongly asymmetric Wilson loops with the smaller extension of the loop limited from above. This equation was then applied to the temporal Wilson loop in Coulomb gauge, keeping only the instantaneous part of the temporal gluon propagator. In this case the resulting Wilsonian potential coincides with the non-Abelian Coulomb potential. Finally, the Dyson equation was used to calculate the spatial Wilson loop in the Hamiltonian approach to Yang-Mills theory in Coulomb gauge. Although from the solution of this equation the Wilsonian potential can be extracted only up to intermediate distances, a strictly linearly rising potential was found when the perturbative potential was subtracted. The emergence of the area law in the Wilson loop found from the static Coulomb gauge propagator is consistent with the perimeter law for the 't Hooft loop found with the wave functional of the variational approach in [24].

An exact Dyson-Schwinger equation for the Wilson loop is given by the so-called loop equation [26]. Like all DysonSchwinger equations this equation is not closed but is part of a tower of integral equations, which need truncations and approximations to arrive at a closed manageable set of equations. Such studies should eventually be carried out. 


\section{Acknowledgments}

The authors are grateful to N. Brambilla, G. Burgio, D. Campagnari, M. Quandt, J. Rafelski, P. Watson and A. V. Zayakin for useful discussions. This work was supported by the Deutsche Forschungsgemeinschaft (DFG) under contract Re856/6-3 and by the Europäisches Graduiertenkolleg "Hadronen im Vakuum, Kernen und Sternen" BaselGraz-Tübingen.

\section{APPENDIX A: THE SCHRÖDINGER POTENTIAL}

The potential (23) in the 1-dimensional Schrödinger equation is, up to a constant prefactor, given by the quantity (9), which is the gluon propagator in coordinate space contracted with the two "temporal" paths $x^{ \pm}(s)$. Below we will calculate this quantity for transversal gluon propagators in arbitrary dimensions $d$.

In Euclidean coordinate space a (translationally invariant) transversal gluon propagator can be expressed by the Fourier integral

$$
D_{\mu \nu}(x)=\int \frac{d^{d} k}{(2 \pi)^{d}}\left(\delta_{\mu \nu}-\hat{k}_{\mu} \hat{k}_{\nu}\right) e^{i k x} \bar{D}\left(k^{2}\right)
$$

where $\hat{k}_{\mu}=k_{\mu} / \sqrt{k^{2}}$ and $\bar{D}\left(k^{2}\right)$ is a function of $k^{2}=k_{\mu} k_{\mu}$ only. Obviously $D_{\mu \nu}(x)$ has the tensor structure

$$
D_{\mu \nu}(x)=\delta_{\mu \nu} I(x)-I_{\mu \nu}(x)
$$

where

$$
I_{\mu \nu}(x)=\int \frac{d^{d} k}{(2 \pi)^{d}} \hat{k}_{\mu} \hat{k}_{\nu} e^{i k x} \bar{D}\left(k^{2}\right)
$$

and $I(x)=I_{\mu \mu}(x)$. Since $x^{\mu}$ is the only external vector, on which $I_{\mu \nu}(x)$ depends, the following tensor decomposition holds

$$
I_{\mu \nu}(x)=\delta_{\mu \nu} I^{1}(x)+\hat{x}_{\mu} \hat{x}_{\nu} I^{2}(x), \hat{x}_{\mu}=\frac{x_{\mu}}{\sqrt{x^{2}}} .
$$

Contracting this equation with $\delta_{\mu \nu}$ and $\hat{x}_{\mu} \hat{x}_{\nu}$ we find

$$
\begin{aligned}
d I^{1}(x)+I^{2}(x) & =I(x) \\
I^{1}(x)+I^{2}(x) & =\bar{I}(x),
\end{aligned}
$$

where $\bar{I}(x)=\hat{x}_{\mu} I_{\mu \nu}(x) \hat{x}_{\nu}$. Solving the last two Eqs. for $I^{1,2}(x)$ yields

$$
\begin{aligned}
& (d-1) I^{1}(x)=I(x)-\bar{I}(x) \\
& (d-1) I^{2}(x)=d \bar{I}(x)-I(x) .
\end{aligned}
$$

Inserting these relations into Eq. (A4) we find for the transversal propagator in coordinate space

$$
(d-1) D_{\mu \nu}(x)=\delta_{\mu \nu}[(d-2) I(x)+\bar{I}(x)]-\hat{x}_{\mu} \hat{x}_{\nu}[d \bar{I}(x)-I(x)] .
$$

The remaining integrals

$$
\left\{\begin{array}{l}
I\left(x^{2}\right) \\
\bar{I}\left(x^{2}\right)
\end{array}\right\}=\int \frac{d^{d} k}{(2 \pi)^{d}} e^{i k \cdot x} \bar{D}\left(k^{2}\right)\left\{\begin{array}{c}
1 \\
(\hat{k} \cdot \hat{x})^{2}
\end{array}\right\}
$$

are worked out in the standard fashion using spherical coordinates in $k$-space and putting the $d$-axis of $k$-space parallel to $x_{\mu}$. The integrals over the first $d-2$ angles are trivial yielding the volume of the unit sphere $S_{d-2}$ in $d-1$ dimensions

$$
\int_{S_{d-2}}=\frac{(d-1) \pi^{\frac{d-1}{2}}}{\Gamma\left(\frac{d+1}{2}\right)} .
$$


The integrals over the last angle yield with $z=\hat{x} \cdot \hat{k}$

$$
\begin{aligned}
\int_{-1}^{1} d z e^{i z k x} & =2 \frac{\sin k x}{k x} \\
\int_{-1}^{1} d z z^{2} e^{i z k x} & =2 \frac{\sin k x}{k x}+\frac{4}{k^{2} x^{2}}\left(\cos k x-\frac{\sin k x}{k x}\right) .
\end{aligned}
$$

Inserting these results into (A9) we obtain

$$
\begin{aligned}
& I(x)=2 C_{d} \int_{0}^{\infty} d k k^{d-1} \bar{D}\left(k^{2}\right) \frac{\sin k x}{k x} \\
& \bar{I}(x)=2 C_{d} \int_{0}^{\infty} d k k^{d-1} \bar{D}\left(k^{2}\right)\left[\frac{\sin k x}{k x}+\frac{2}{k^{2} x^{2}}\left(\cos k x-\frac{\sin k x}{k x}\right)\right],
\end{aligned}
$$

where

$$
C_{d}=\frac{(d-1) \pi^{\frac{d-1}{2}}}{(2 \pi)^{d} \Gamma\left(\frac{d+1}{2}\right)} .
$$

To work out the remaining one-dimensional integrals requires the explicit form of the scalar function $\bar{D}\left(k^{2}\right)$. For definiteness we consider a planar temporal Wilson loop in the 0-1-plane and choose the parametrization of the temporal pieces as

$$
x_{\mu}^{ \pm}(s)=\left(s, \pm \frac{L}{2}, 0, \ldots\right)
$$

For these paths we have

$$
\dot{x}_{\mu}^{ \pm}(s)=(1,0,0, \ldots)=: \hat{e}_{\mu}
$$

and

$$
x_{\mu}:=x_{\mu}^{+}(S)-x_{\mu}^{-}(T)=L(r, 1,0,0, \ldots)
$$

so that

$$
x^{2}=L^{2}\left(1+r^{2}\right)
$$

and

$$
(\hat{x} \cdot \hat{e})^{2}=\frac{r^{2}}{1+r^{2}} .
$$

With these results we eventually find for the quantity (9) from (A12)

$$
(d-1) D\left(x^{2}\right)=\left[(d-2) I\left(x^{2}\right)+\bar{I}\left(x^{2}\right)\right]-\frac{r^{2}}{1+r^{2}}\left[d \bar{I}\left(x^{2}\right)-I\left(x^{2}\right)\right]
$$

where $x^{2}$ is given by Eq. (A17).

[1] A. Cucchieri and D. Zwanziger, Phys. Rev. D 65, 014002 (2002) arXiv:hep-th/0008248.

[2] N. H. Christ and T. D. Lee, Phys. Rev. D 22, 939 (1980) [Phys. Scripta 23, 970 (1981)].

[3] D. Zwanziger, Phys. Rev. Lett. 90, 102001 (2003) arXiv:hep-lat/0209105. 
[4] L. von Smekal, A. Hauck and R. Alkofer, Annals Phys. 267, 1 (1998) [Erratum-ibid. 269, 182 (1998)] arXiv:hep-ph/9707327. C. S. Fischer, J. Phys. G 32, R253 (2006) arXiv:hep-ph/0605173.

[5] P. Watson and H. Reinhardt, Phys. Rev. D 75, 045021 (2007) arXiv:hep-th/0612114.

[6] A. P. Szczepaniak and E. S. Swanson, Phys. Rev. D 65, 025012 (2002) arXiv:hep-ph/0107078. A. P. Szczepaniak, Phys. Rev. D 69, 074031 (2004) arXiv:hep-ph/0306030.

[7] C. Feuchter and H. Reinhardt, Phys. Rev. D 70, 105021 (2004) arXiv:hep-th/0408236]. C. Feuchter and H. Reinhardt, arXiv:hep-th/0402106

[8] H. Reinhardt and C. Feuchter, Phys. Rev. D 71, 105002 (2005) arXiv:hep-th/0408237.

[9] D. Epple, H. Reinhardt and W. Schleifenbaum, Phys. Rev. D 75, 045011 (2007) arXiv:hep-th/0612241.

[10] D. Epple, H. Reinhardt, W. Schleifenbaum and A. P. Szczepaniak, Phys. Rev. D 77, 085007 (2008) arXiv:0712.3694 [hep-th]].

[11] V. N. Gribov, Nucl. Phys. B 139, 1 (1978).

[12] D. Zwanziger, Nucl. Phys. B 378, 525 (1992).

[13] J. K. Erickson, G. W. Semenoff, R. J. Szabo and K. Zarembo, Phys. Rev. D 61, 105006 (2000) arXiv:hep-th/9911088. J. K. Erickson, G. W. Semenoff and K. Zarembo, Nucl. Phys. B 582, 155 (2000) arXiv:hep-th/0003055.

[14] A. V. Zayakin and J. Rafelski, Phys. Rev. D 80, 034024 (2009) arXiv:0905.2317 [hep-ph]].

[15] J. Greensite, Prog. Part. Nucl. Phys. 51, 1 (2003) arXiv:hep-lat/0301023.

[16] D. Zwanziger, Phys. Rev. D 70, 094034 (2004) arXiv:hep-ph/0312254.

[17] S. D. Drell, SLAC-PUB-2694 (1981).

[18] D. R. Campagnari, H. Reinhardt and A. Weber, Phys. Rev. D 80, 025005 (2009) arXiv:0904.3490 [hep-th]].

[19] P. Watson and H. Reinhardt, Phys. Rev. D 76, 125016 (2007) arXiv:0709.0140 [hep-th]]. P. Watson and H. Reinhardt, Phys. Rev. D 77, 025030 (2008) arXiv:0709.3963 [hep-th]].

[20] A. Cucchieri and D. Zwanziger, Nucl. Phys. Proc. Suppl. 119, 727 (2003) arXiv:hep-lat/0209068.

[21] J. Greensite and S. Olejnik, Phys. Rev. D 67, 094503 (2003) arXiv:hep-lat/0302018.

[22] K. Langfeld and L. Moyaerts, Phys. Rev. D 70, 074507 (2004) arXiv:hep-lat/0406024].

[23] G. Burgio, M. Quandt and H. Reinhardt, Phys. Rev. Lett. 102, 032002 (2009) [arXiv:0807.3291 [hep-lat]].

[24] H. Reinhardt and D. Epple, Phys. Rev. D 76, 065015 (2007) arXiv:0706.0175 [hep-th]].

[25] W. Schleifenbaum, PhD thesis, Universität Tübingen (2008) arXiv:0809.1339 [hep-th]].

[26] A. M. Polyakov, Gauge fields and strings, Harwood Academic Publishers, Chur, 1987. 